\title{
Site of Pulmonary Resistance in Cor Pulmonale in Chronic Bronchitis
}

\author{
F. HERLES, V. JEŽEK, AND S. DAUM \\ From Second Medical Clinic, Charles University, Prague, Czechoslovakia
}

The pathogenesis of pulmonary hypertension in chronic bronchitis is still obscure, though the relation of pulmonary artery pressure and tension of alveolar gases has been recognized since the classical experiment of von Euler and Liljestrand (1946).

Our interest in the site of resistance was aroused by the increased pulmonary artery wedge pressure in cor pulmonale without overt pathological changes in the left heart. Most of these patients were middle-aged or older men and ischaemic heart disease was suspected. However, the coincidence of ischaemic heart disease with cor pulmonale was found to be less frequent than would correspond to their separate incidence in the general population (Králová et al., 1965).

Pulmonary artery wedge pressure is virtually an artefact and the result of interplay of several factors (Herles, 1966). Analysing a large group of patients with chronic obstructive bronchitis, emphysema, and cor pulmonale, we have attempted to elucidate the origin of increases in pulmonary artery wedge pressure as well as the site of resistance modifying the pressure gradient in the pulmonary circulation.

\section{SUbJeCtS AND MethodS}

Our subjects comprised a group of 121 patients with chronic bronchitis and pulmonary emphysema. No patients with other forms of pneumopathy or with vascular forms of cor pulmonale were included in the series.

Catheterization of the right heart and the pulmonary artery was carried out in all patients in the morning, after food, in the supine position. Blood pressure was taken by the Hellige tensometer with the zero level $10 \mathrm{~cm}$. above the catheterization table and recorded by the Multiscriptor Hellige. Another catheter was placed in the femoral artery. During the investigation, the patient breathed atmospheric air through the Metabograph (Fleisch). After reaching the steady state, blood

Received November 24, 1967. for gas analysis was taken from the femoral and pulmonary artery under strict anaerobic conditions.

The $\mathrm{O}_{2}$ and $\mathrm{CO}_{2}$ content of the blood was determined by the method of Van Slyke and Neill (1924), the blood $p \mathrm{H}$ by the $p \mathrm{H}$ meter (Godart and Cambridge). The blood $\mathrm{CO}_{2}$ tension was calculated from the $\mathrm{CO}_{2}$ content and $\mathrm{pH}$.

Cardiac output was determined from oxygen consumption and the arterio-venous oxygen difference using the Fick's formula. Total pulmonary resistance and arteriolar resistance were expressed in arbitrary Wood's units calculated according to the following formulae.

$$
\mathrm{TPR}=\frac{\text { mPAP }}{\mathrm{CO}} \quad \text { PAR }=\frac{(\mathrm{mPAP}-\mathrm{mPAWP})}{\mathrm{CO}} .
$$

The work of the right ventricle, expressed in kg.m./, $\mathrm{min} . / \mathrm{m} .{ }^{2} \mathrm{BSA}$, was computed from the formula

$$
\mathrm{W}_{\mathrm{r}}=\frac{\mathrm{CI} \times 1.055 \times 13.6(\mathrm{mPAP}-\mathrm{mRAP})}{1000},
$$

where $\mathrm{mRAP}$ is mean pressure in the right atrium, mPAP is mean pressure in the pulmonary artery, mPAWP is mean pressure in the wedged pulmonary artery, $\mathrm{CO}$ is cardiac output in $1 . / \mathrm{min}$., $\mathrm{CI}$ is cardiac index, TPR is total pulmonary resistance, PAR is pulmonary arteriolar resistance, and $W_{r}$ is work of the right ventricle.

Extra-arteriolar or post-arteriolar (EAPR) pulmonary resistance was calculated from the difference between total resistance and arterial resistance. In calculating total pulmonary resistance, the left atrial pressure was not deducted, since it had not been ascertained and the deduction of an arbitrary value of $5 \mathrm{~mm}$. Hg does not increase the comparability of the figures. Extraarteriolar resistance includes both the resistances of pulmonary venules and the "resistance" of the left side of the heart.

Pressures at rest in the right atrium exceeding $5 \mathrm{~mm}$. $\mathrm{Hg}$, in the pulmonary artery exceeding $20 \mathrm{~mm} . \mathrm{Hg}$, and in the wedged pulmonary artery reaching or exceeding $12 \mathrm{~mm} . \mathrm{Hg}$, were considered to be increased.

To follow separately the influence of pulmonary hypertension and of heart failure, the group of 92 patients with cor pulmonale was subdivided into 3 groups: (1) 45 patients who at the time of examination and for two 
TABLE I

HAEMODYNAMIC AND RESPIRATORY FINDINGS IN CHRONIC BRONCHITIS WITHOUT PULMONARY HYPERTENSION (CONTROLS)

\begin{tabular}{|c|c|c|c|c|c|c|c|c|c|c|c|}
\hline Case No. & $\underset{(\mathbf{m m} \cdot \mathbf{H g})}{\mathbf{m R A P}}$ & $\underset{(\mathbf{m m} \cdot \mathbf{H} \mathbf{g})}{\mathbf{m P A}}$ & $\begin{array}{l}\text { mPAWP } \\
(\mathbf{m m} . \mathbf{H g})\end{array}$ & $\begin{array}{c}\text { Cardiac } \\
\text { index } \\
\left(1 . / \mathrm{min} . / \mathrm{m} .^{2}\right)\end{array}$ & $\begin{array}{l}\text { Total } \\
\text { pulm. } \\
\text { resist. } \\
\text { (units) }\end{array}$ & $\underset{\text { (units) }}{\mathbf{P A R}}$ & $\begin{array}{l}\text { EAPR } \\
\text { (units) }\end{array}$ & $\underset{\substack{\text { m. } \\
(\mathbf{k})}}{\mathrm{W}_{\mathbf{r}}}$ & $\underset{(\%)}{\mathrm{S}_{\mathrm{a}} \mathrm{O}_{2}}$ & $\mid \underset{\left(\mathbf{m m} . \mathbf{H}_{\mathbf{g}}\right)}{\mathbf{P}_{\mathbf{a}} \mathbf{C O}_{\mathbf{g}}}$ & pH \\
\hline $\begin{array}{r}1 \\
2 \\
3 \\
4 \\
5 \\
6 \\
7 \\
8 \\
9 \\
10 \\
11 \\
12 \\
13 \\
14 \\
15 \\
16 \\
17 \\
18 \\
19 \\
20 \\
21 \\
22 \\
23 \\
24 \\
25 \\
26 \\
27 \\
28 \\
29\end{array}$ & $\begin{array}{l}4 \cdot 6 \\
3 \cdot 0 \\
2 \cdot 0 \\
1 \cdot 0 \\
1 \cdot 0 \\
1 \cdot 0 \\
1 \cdot 5 \\
2 \cdot 0 \\
1 \cdot 2 \\
3 \cdot 0 \\
1 \cdot 0 \\
0 \cdot 0 \\
5 \cdot 0 \\
5 \cdot 4 \\
0 \cdot 3 \\
4 \cdot 8 \\
4 \cdot 7 \\
0 \cdot 0 \\
4 \cdot 8 \\
2 \cdot 0 \\
2 \cdot 0 \\
2 \cdot 0 \\
1.5 \\
1 \cdot 4 \\
4 \cdot 0 \\
4 \cdot 8 \\
0 \cdot 0\end{array}$ & $\begin{array}{l}18 \\
17 \\
18 \\
16 \\
11 \\
12 \\
15 \\
13 \\
15 \\
16 \\
14 \\
16 \\
14 \\
12 \\
11 \\
8 \\
15 \\
17 \\
15 \\
17 \\
16 \\
13 \\
15 \\
17 \\
14 \\
16 \\
13 \\
16 \\
12\end{array}$ & 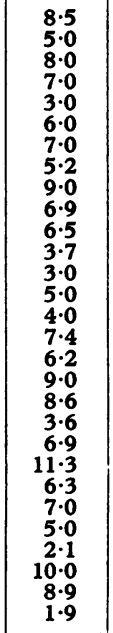 & $\begin{array}{l}2 \cdot 8 \\
2 \cdot 7 \\
5 \cdot 6 \\
3 \cdot 7 \\
3 \cdot 6 \\
2 \cdot 0 \\
3 \cdot 3 \\
2 \cdot 3 \\
3 \cdot 5 \\
4 \cdot 1 \\
4 \cdot 0 \\
3 \cdot 0 \\
4 \cdot 6 \\
3 \cdot 6 \\
4 \cdot 2 \\
2 \cdot 1 \\
3 \cdot 4 \\
2 \cdot 6 \\
3 \cdot 4 \\
5 \cdot 5 \\
4.3 \\
2.9 \\
3 \cdot 6 \\
3 \cdot 8 \\
2 \cdot 8 \\
5 \cdot 8 \\
2 \cdot 2 \\
3 \cdot 1 \\
2 \cdot 5\end{array}$ & $\begin{array}{l}3.3 \\
3.7 \\
1.6 \\
2.4 \\
1.8 \\
3.8 \\
2.6 \\
2.8 \\
2.3 \\
2.2 \\
2.1 \\
2.9 \\
1 \cdot 7 \\
2.2 \\
1.3 \\
2.1 \\
1.7 \\
3.3 \\
2.5 \\
1.8 \\
2.3 \\
2.3 \\
2.5 \\
2.4 \\
2.6 \\
1.6 \\
3.3 \\
2.7 \\
2.9\end{array}$ & $\begin{array}{l}1.8 \\
2.6 \\
0.9 \\
1.4 \\
1.3 \\
1.9 \\
1.4 \\
1.7 \\
0.9 \\
1.3 \\
1.1 \\
2.2 \\
1.3 \\
1.5 \\
0.8 \\
0.5 \\
1.0 \\
1.3 \\
1.1 \\
1.4 \\
1.3 \\
0.3 \\
1.3 \\
1.4 \\
1.7 \\
1.3 \\
0.7 \\
1.4 \\
2.4\end{array}$ & $\begin{array}{l}1.5 \\
1.1 \\
0.7 \\
1.0 \\
0.5 \\
1.9 \\
1.2 \\
1.1 \\
1.4 \\
0.9 \\
1.0 \\
0.7 \\
0.4 \\
0.7 \\
0.5 \\
1.6 \\
0.7 \\
2.0 \\
1.4 \\
0.4 \\
1.0 \\
2.0 \\
1.2 \\
1.0 \\
0.9 \\
0.3 \\
2.6 \\
1.3 \\
0.5\end{array}$ & $\begin{array}{l}0.64 \\
0.54 \\
1.29 \\
0.79 \\
0.32 \\
0.66 \\
0.40 \\
0.64 \\
0.89 \\
0.64 \\
0.65 \\
0.92 \\
0.84 \\
0.33 \\
0.25 \\
0.44 \\
0.50 \\
1.36 \\
0.58 \\
0.45 \\
0.64 \\
0.82 \\
0.51 \\
1.13 \\
0.29 \\
0.51 \\
0.43\end{array}$ & $\begin{array}{l}92 \\
98 \\
97 \\
82 \\
86 \\
88 \\
87 \\
90 \\
87 \\
87 \\
87 \\
76 \\
90 \\
90 \\
91 \\
87 \\
77 \\
88 \\
86 \\
88 \\
84 \\
83 \\
91 \\
89 \\
81 \\
90\end{array}$ & $\begin{array}{l}44 \\
= \\
= \\
= \\
= \\
\overline{-} \\
42 \\
38 \\
39 \\
37 \\
25 \\
35 \\
42 \\
35 \\
41 \\
37 \\
27 \\
37 \\
33 \\
38 \\
39 \\
40 \\
47 \\
39 \\
39 \\
39\end{array}$ & $\begin{array}{l}7 \cdot 33 \\
= \\
= \\
= \\
= \\
= \\
7 \cdot 37 \\
7 \cdot 41 \\
7 \cdot 43 \\
7 \cdot 39 \\
7 \cdot 44 \\
7 \cdot 41 \\
7 \cdot 35 \\
7 \cdot 40 \\
7 \cdot 40 \\
7 \cdot 40 \\
7 \cdot 40 \\
7 \cdot 38 \\
7 \cdot 41 \\
7 \cdot 39 \\
7 \cdot 42 \\
7 \cdot 39 \\
7 \cdot 36 \\
7 \cdot 39 \\
7 \cdot 37 \\
7 \cdot 35\end{array}$ \\
\hline $\begin{array}{c}\text { Total: Average } \\
\text { Sigma }\end{array}$ & $\begin{array}{l}2 \cdot 0 \\
1 \cdot 3\end{array}$ & $\begin{array}{r}14 \cdot 5 \\
2 \cdot 7\end{array}$ & $\begin{array}{l}6 \cdot 3 \\
2 \cdot 4\end{array}$ & $\begin{array}{l}3.58 \\
1.4\end{array}$ & $\begin{array}{l}2.48 \\
0.8\end{array}$ & $\begin{array}{l}1 \cdot 34 \\
0.5\end{array}$ & $\begin{array}{l}1 \cdot 14 \\
0.6\end{array}$ & $\begin{array}{l}0.64 \\
0 \cdot 34\end{array}$ & $\begin{array}{r}87 \cdot 8 \\
6 \cdot 3\end{array}$ & $\begin{array}{r}37.6 \\
5.9\end{array}$ & $\begin{array}{l}7 \cdot 393 \\
0 \cdot 08\end{array}$ \\
\hline
\end{tabular}

months before examination had not had any clinical signs of right heart failure and whose right atrial pressure was normal. (2) 29 patients with clinical signs of right heart failure at the time of examination. On catheterization the pressure in the right atrium was raised. (3) 18 patients who on admission to the department also had signs of failure of the right heart, but who had already been treated and whose clinical condition was improved.

A separate group of 29 patients with chronic obstructive bronchitis without pulmonary hypertension was used as controls.

The data of the individual groups and their differences were evaluated by the Student $t$ test. Correlation coefficients were calculated according to Pearson and Spearman.

\section{Results and Their ANALysis}

Chronic Obstructive Bronchitis Without Pulmonary Hypertension (Controls). (Table I). In chronic bronchitis without hypertension in the pulmonary artery (mean pulmonary artery pressure averaging $14.5 \mathrm{~mm} . \mathrm{Hg}$ ) the pressure in the right atrium was always normal and so was the pulmonary artery wedge pressure. Blood flow, calculated resistance, and the work of the right ventricle were also within normal limits. There was a moderately severe hypoxaemia, normal or slightly decreased $\mathrm{CO}_{2}$ tension, and a normal $p H$. These patients were considered controls for comparison with chronic cor pulmonale.

Cor Pulmonale Without Heart Failure (Compensated) (Table II). The mean pressure in the right atrium was higher than in the previous group, but still within normal range. The difference was not statistically significant $(t=1.016, p>0 \cdot 10)$. The mean pressure in the pulmonary artery was increased, and the pressure in the wedged pulmonary artery did not differ significantly from that in controls $(p>0 \cdot 10)$, though in some cases it was found to be higher.

The cardiac output and cardiac index remained the same as in simple chronic bronchitis. Total and arteriolar resistance were significantly increased $(p<0.01)$, but the extra-arteriolar resistance remained the same as in the previous group on the average. Neither was the arterial oxygen saturation significantly decreased $(p>0 \cdot 10)$ nor was the difference in the arterial $p H$ significant. However, the increase in $\mathrm{CO}_{2}$ tension in the arterial blood was statistically significant $(p<0.05)$.

Cor Pulmonale in Right Heart Failure (Decompensated) (Table III). The difference from the controls was most significant in this group. The 
TABLE II

HAEMODYNAMIC AND RESPIRATORY FINDINGS IN COR PULMONALE WITHOUT HEART FAILURE

\begin{tabular}{|c|c|c|c|c|c|c|c|c|c|c|c|}
\hline Case. No. & $\underset{(\mathrm{mm} . \mathrm{Hg})}{\operatorname{mRAP}}$ & $\underset{(\mathbf{m m} . \mathbf{H g})}{\operatorname{mPAP}}$ & $\begin{array}{l}\text { mPAWP } \\
(\mathbf{m m . H g})\end{array}$ & $\begin{array}{c}\text { Cardiac } \\
\text { index } \\
\left(1 . / \mathrm{min} . / \mathrm{m} \cdot .^{2}\right)\end{array}$ & $\begin{array}{l}\text { Total } \\
\text { pulm. } \\
\text { resist. } \\
\text { (units) }\end{array}$ & $\underset{\text { (units) }}{\text { PAR }}$ & $\begin{array}{l}\text { EAPR } \\
\text { (units) }\end{array}$ & $\underset{\substack{\mathrm{m} . \\
.^{2}}}{\mathrm{~W}_{\mathrm{r}}}$ & $\underset{(\%)}{\mathrm{S}_{\mathrm{a}} \mathrm{O}_{2}}$ & $\mid \begin{array}{c}\mathbf{P}_{\mathrm{g}} \mathrm{CO}_{2} \\
\left(\mathrm{~mm} . \mathrm{H}_{\mathrm{g}}\right)\end{array}$ & $p H$ \\
\hline $\begin{array}{r}1 \\
2 \\
3 \\
4 \\
5 \\
6 \\
7 \\
8 \\
9 \\
10 \\
11 \\
12 \\
13 \\
14 \\
15 \\
16 \\
17 \\
18 \\
19 \\
20 \\
21 \\
22 \\
23 \\
24 \\
25 \\
26 \\
27 \\
28 \\
29 \\
30 \\
31 \\
32 \\
33 \\
34 \\
35 \\
36 \\
37 \\
38 \\
39 \\
40 \\
41 \\
42 \\
43 \\
44 \\
45\end{array}$ & $\begin{array}{l}4.8 \\
3.8 \\
0.8 \\
4.1 \\
1.8 \\
3.6 \\
0.0 \\
1.5 \\
2.0 \\
2.0 \\
2.0 \\
1.0 \\
1.0 \\
2.0 \\
3.0 \\
2.0 \\
2.3 \\
1.0 \\
1.7 \\
1.8 \\
2.0 \\
2.0 \\
2.0 \\
4.0 \\
2.0 \\
1.4 \\
3.0 \\
3.9 \\
1.2 \\
3.5 \\
5.0 \\
1.0 \\
3.0 \\
2.0 \\
5.0 \\
4.0 \\
6.0 \\
2.0 \\
9.2 \\
7.0 \\
0.0 \\
-1 \\
2.3 \\
-1 \\
\end{array}$ & $\begin{array}{l}30 \\
32 \\
20 \\
24 \\
26 \\
25 \\
21 \\
41 \\
22 \\
23 \\
26 \\
27 \\
22 \\
32 \\
35 \\
24 \\
31 \\
23 \\
26 \\
30 \\
38 \\
40 \\
37 \\
27 \\
32 \\
27 \\
41 \\
26 \\
21 \\
22 \\
19 \\
28 \\
19 \\
20 \\
21 \\
36 \\
19 \\
23 \\
28 \\
22 \\
47 \\
34 \\
21 \\
35 \\
23\end{array}$ & $\begin{array}{r}7.5 \\
15.0 \\
2.7 \\
3.5 \\
4.6 \\
7.3 \\
0.0 \\
12.4 \\
8.0 \\
7.0 \\
9.0 \\
9.0 \\
2.0 \\
2.0 \\
5.0 \\
2.0 \\
5.0 \\
7.0 \\
5.0 \\
7.0 \\
7.0 \\
6.0 \\
6.0 \\
9.0 \\
16.0 \\
13.0 \\
17.0 \\
8.9 \\
5.7 \\
5.7 \\
8.0 \\
6.2 \\
2.3 \\
3.0 \\
5.0 \\
5.0 \\
11.0 \\
6.0 \\
14.0 \\
13.0 \\
3.0 \\
10.5 \\
3.2 \\
11.5 \\
5.0\end{array}$ & $\begin{array}{l}3 \cdot 0 \\
4 \cdot 4 \\
5 \cdot 8 \\
4 \cdot 6 \\
3 \cdot 6 \\
3 \cdot 3 \\
4 \cdot 0 \\
2 \cdot 7 \\
5 \cdot 2 \\
2 \cdot 5 \\
2 \cdot 5 \\
4 \cdot 3 \\
3 \cdot 0 \\
3 \cdot 8 \\
4 \cdot 1 \\
2 \cdot 0 \\
3 \cdot 8 \\
2 \cdot 8 \\
3 \cdot 7 \\
3 \cdot 8 \\
3 \cdot 6 \\
4 \cdot 0 \\
3 \cdot 4 \\
5 \cdot 0 \\
5 \cdot 0 \\
4 \cdot 6 \\
2 \cdot 6 \\
2 \cdot 5 \\
2 \cdot 4 \\
3 \cdot 5 \\
2 \cdot 1 \\
7 \cdot 1 \\
5 \cdot 7 \\
4 \cdot 7 \\
4 \cdot 7 \\
3 \cdot 8 \\
5 \cdot 0 \\
3 \cdot 5 \\
3 \cdot 2 \\
3 \cdot 6 \\
4 \cdot 2 \\
3 \cdot 9 \\
3 \cdot 1 \\
3 \cdot 3 \\
3 \cdot 4\end{array}$ & 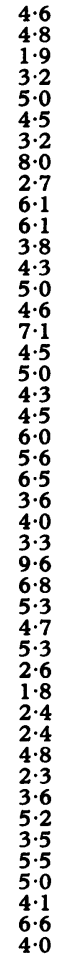 & $\begin{array}{l}3 \cdot 9 \\
2 \cdot 6 \\
1 \cdot 6 \\
2 \cdot 9 \\
4 \cdot 1 \\
3 \cdot 3 \\
3 \cdot 2 \\
5 \cdot 6 \\
1 \cdot 7 \\
4 \cdot 2 \\
4 \cdot 0 \\
2 \cdot 5 \\
3 \cdot 9 \\
4 \cdot 7 \\
3 \cdot 9 \\
6 \cdot 5 \\
3 \cdot 7 \\
3 \cdot 5 \\
3 \cdot 5 \\
3 \cdot 5 \\
4 \cdot 9 \\
4 \cdot 7 \\
5 \cdot 4 \\
2 \cdot 4 \\
2 \cdot 0 \\
1 \cdot 7 \\
5 \cdot 8 \\
4 \cdot 5 \\
3 \cdot 9 \\
3 \cdot 7 \\
3 \cdot 1 \\
2 \cdot 0 \\
1 \cdot 6 \\
2 \cdot 1 \\
1 \cdot 8 \\
4 \cdot 1 \\
1 \cdot 0 \\
2 \cdot 7 \\
2 \cdot 6 \\
1 \cdot 4 \\
5 \cdot 2 \\
3 \cdot 2 \\
3 \cdot 5 \\
4 \cdot 4 \\
3 \cdot 1\end{array}$ & $\begin{array}{l}0.7 \\
2.2 \\
0.3 \\
0.3 \\
0.9 \\
1.2 \\
0.0 \\
2.4 \\
1.0 \\
1.9 \\
2.1 \\
1.3 \\
0.4 \\
0.3 \\
0.7 \\
0.6 \\
0.8 \\
1.5 \\
0.8 \\
1.0 \\
1.1 \\
0.9 \\
1.1 \\
1.2 \\
2.0 \\
1.6 \\
3.8 \\
2.3 \\
1.4 \\
1.0 \\
2.2 \\
0.6 \\
0.2 \\
0.3 \\
0.6 \\
0.7 \\
1.3 \\
0.9 \\
2.6 \\
2.1 \\
0.3 \\
1.8 \\
0.6 \\
2.2 \\
0.9 \\
\end{array}$ & 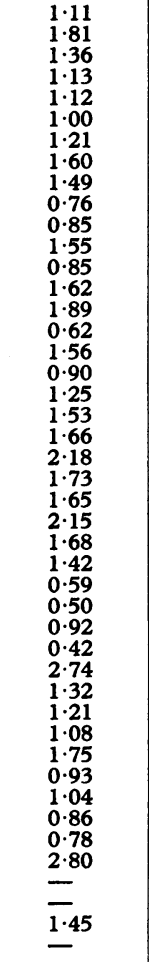 & 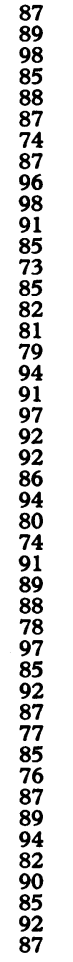 & $\begin{array}{l}44 \\
45 \\
59 \\
55 \\
47 \\
52 \\
62 \\
= \\
= \\
= \\
= \\
= \\
= \\
= \\
= \\
= \\
= \\
= \\
= \\
= \\
= \\
37 \\
30 \\
52 \\
40 \\
39 \\
31 \\
\overline{4} \\
43 \\
38 \\
42 \\
37 \\
37 \\
43 \\
44\end{array}$ & $\begin{array}{l}7 \cdot 37 \\
7 \cdot 33 \\
7 \cdot 28 \\
7 \cdot 35 \\
7 \cdot 40 \\
7 \cdot 36 \\
7 \cdot 29 \\
= \\
= \\
= \\
= \\
= \\
= \\
= \\
= \\
= \\
= \\
= \\
= \\
= \\
= \\
77 \cdot 41 \\
7 \cdot 37 \\
7 \cdot 36 \\
7 \cdot 39 \\
7 \cdot 39 \\
7 \cdot 41 \\
= \\
7 \cdot 35 \\
7 \cdot 35 \\
7 \cdot 38 \\
7 \cdot 37 \\
7 \cdot 35 \\
7 \cdot 35 \\
7 \cdot 36 \\
7 \cdot 38\end{array}$ \\
\hline $\begin{array}{c}\text { Total: Average } \\
\text { Sigma }\end{array}$ & $\begin{array}{l}2 \cdot 7 \\
2 \cdot 1\end{array}$ & $\begin{array}{r}27 \cdot 7 \\
4 \cdot 1\end{array}$ & $\begin{array}{l}7 \cdot 2 \\
4 \cdot 9\end{array}$ & $\begin{array}{l}3 \cdot 81 \\
1.55\end{array}$ & $\begin{array}{l}4 \cdot 61 \\
1 \cdot 3\end{array}$ & $\begin{array}{l}3 \cdot 40 \\
1 \cdot 3\end{array}$ & $\begin{array}{l}1 \cdot 21 \\
0 \cdot 8\end{array}$ & $\begin{array}{l}1 \cdot 31 \\
0 \cdot 51\end{array}$ & $\begin{array}{r}86 \cdot 9 \\
8 \cdot 1\end{array}$ & $\begin{array}{r}43 \cdot 6 \\
6 \cdot 2\end{array}$ & $\begin{array}{l}7 \cdot 362 \\
0.05\end{array}$ \\
\hline
\end{tabular}

mean pressure in the right atrium was significantly raised $(\mathrm{p}<0.001)$ as compared with the two previous groups, as was the mean pressure in the pulmonary artery $(p<0.01)$. The increase in the pulmonary artery wedge pressure was highly significant, the average value being almost twice that of the preceding groups. This pressure was found to be increased in more than half of the decompensated patients. The blood flow values were not statistically different from those of the preceding groups $(p>0 \cdot 10)$. However, the increase in the mean value of total, arteriolar, and extra-arteriolar pulmonary resistances was highly significant $(p<0.01$, for EAPR $p<0.001)$. There was a statistically significant increase in the work of the right ventricle $(p<0.01)$ as opposed to the control group, but when compared with the compensated cor pulmonale group, the difference was not significant because of the wide scatter of the results $(p>0.05)$. When compared with this group there was a significant decrease in the oxygen saturation $(\mathrm{p}<0.05)$, a highly significant increase in $\mathrm{CO}_{2}$ tension in the arterial blood $(p<0.01)$, and a significant decrease in the $p \mathrm{H}(\mathrm{p}<0.05)$.

Cor Pulmonale after Recovery From Failure (Recompensated) (Table IV). Haemodynamic values and blood gas readings were within the range of mean findings in cor pulmonale with and without heart failure. Therefore, statistical differences among these groups were not significant, though in some parameters, e.g. extra-arteriolar resistance, work of the right ventricle, and oxygen saturation of the arterial blood, this group resembled that with heart failure.

Interrelation Between Haemodynamic Values and Blood Gases in Individual Groups of Patients. There 
TABLE III

HAEMODYNAMIC AND RESPIRATORY FINDINGS IN COR PULMONALE WITH HEART FAILURE

\begin{tabular}{|c|c|c|c|c|c|c|c|c|c|c|c|}
\hline Case No. & $|\underset{(\mathbf{m m} . \mathbf{H g})}{\operatorname{mRAP}}|$ & $\underset{(\mathbf{m m} \cdot \mathbf{H g})}{\operatorname{mPAP}}$ & $\begin{array}{c}\text { mPAWP } \\
(\mathbf{m m} . \mathbf{H g})\end{array}$ & $\begin{array}{c}\text { Cardiac } \\
\text { index } \\
\left(1 . / \mathrm{min} . / \mathrm{m} .^{2}\right)\end{array}$ & $\begin{array}{l}\text { Total } \\
\text { pulm. } \\
\text { resist. } \\
\text { (units) }\end{array}$ & $\underset{\text { (units) }}{\text { PAR }}$ & $\begin{array}{l}\text { EAPR } \\
\text { (units) }\end{array}$ & $\underset{\substack{\text { m.2) } \\
\text { (kp.m. }}}{W_{r}}$ & $\underset{(\%)}{\mathrm{S}_{\mathrm{a}} \mathrm{O}_{2}}$ & $\underset{\left(\mathbf{m m} . \mathrm{Hg}_{\mathbf{g}}\right)}{\mathbf{P}_{\mathrm{a}} \mathrm{CO}_{\mathbf{2}}}$ & $p \mathbf{H}$ \\
\hline $\begin{array}{r}1 \\
2 \\
3 \\
4 \\
5 \\
6 \\
7 \\
8 \\
9 \\
10 \\
11 \\
12 \\
13 \\
14 \\
15 \\
16 \\
17 \\
18 \\
19 \\
20 \\
21 \\
22 \\
23 \\
24 \\
25 \\
26 \\
27 \\
28 \\
29\end{array}$ & $\begin{array}{r}14 \cdot 4 \\
6 \cdot 8 \\
9.6 \\
11.8 \\
9 \cdot 0 \\
10.0 \\
12.0 \\
8.0 \\
10.0 \\
6.5 \\
7.0 \\
15.0 \\
8.0 \\
10.0 \\
6.0 \\
5.0 \\
7.0 \\
6.0 \\
6.0 \\
26.8 \\
7.8 \\
10.2 \\
5.2 \\
9.5 \\
8.5 \\
8.3 \\
2.5 \\
=\end{array}$ & $\begin{array}{l}66 \\
38 \\
42 \\
48 \\
40 \\
51 \\
24 \\
29 \\
36 \\
42 \\
72 \\
59 \\
41 \\
42 \\
42 \\
32 \\
36 \\
24 \\
39 \\
70 \\
40 \\
27 \\
23 \\
40 \\
49 \\
24 \\
24 \\
31 \\
54\end{array}$ & $\begin{array}{r}20.2 \\
11 \cdot 2 \\
9.0 \\
15.6 \\
21.0 \\
15.0 \\
18.0 \\
15.0 \\
25.0 \\
13.0 \\
25 \cdot 0 \\
17.0 \\
15.0 \\
14.0 \\
7.0 \\
8.0 \\
4.0 \\
9.0 \\
7.0 \\
21.0 \\
10.8 \\
15.8 \\
6.5 \\
8.0 \\
17.0 \\
15.0 \\
7.2 \\
3.5 \\
4.0\end{array}$ & $\begin{array}{l}6 \cdot 2 \\
3 \cdot 6 \\
3 \cdot 6 \\
4 \cdot 0 \\
4 \cdot 7 \\
4 \cdot 1 \\
2 \cdot 3 \\
1 \cdot 7 \\
2 \cdot 7 \\
2 \cdot 2 \\
2 \cdot 8 \\
2 \cdot 6 \\
2 \cdot 8 \\
1 \cdot 8 \\
4 \cdot 1 \\
3 \cdot 1 \\
2 \cdot 3 \\
2 \cdot 0 \\
4 \cdot 7 \\
3 \cdot 4 \\
2 \cdot 8 \\
4 \cdot 7 \\
4 \cdot 0 \\
2 \cdot 9 \\
6 \cdot 5 \\
3 \cdot 3 \\
3 \cdot 6 \\
4 \cdot 2 \\
3 \cdot 4\end{array}$ & 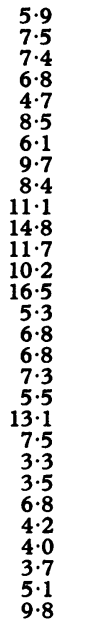 & $\begin{array}{r}4 \cdot 1 \\
4 \cdot 7 \\
5 \cdot 8 \\
4 \cdot 6 \\
2 \cdot 2 \\
6 \cdot 0 \\
1 \cdot 5 \\
4 \cdot 7 \\
3 \cdot 0 \\
7 \cdot 7 \\
9 \cdot 9 \\
8 \cdot 3 \\
6 \cdot 4 \\
11 \cdot 0 \\
4 \cdot 4 \\
5 \cdot 1 \\
6 \cdot 0 \\
4 \cdot 5 \\
4 \cdot 5 \\
9 \cdot 1 \\
5 \cdot 5 \\
1 \cdot 4 \\
2.5 \\
5 \cdot 4 \\
2 \cdot 8 \\
1.5 \\
2.6 \\
4.6 \\
9 \cdot 1\end{array}$ & $\begin{array}{l}1.8 \\
2.8 \\
1.6 \\
2.2 \\
2.5 \\
2.5 \\
4.6 \\
5.0 \\
5.4 \\
3.4 \\
4.9 \\
3.4 \\
3.8 \\
5.5 \\
0.9 \\
1.7 \\
0.8 \\
2.8 \\
1.0 \\
4.0 \\
2.0 \\
1.9 \\
1.0 \\
1.4 \\
1.4 \\
2.5 \\
1.1 \\
0.5 \\
0.7\end{array}$ & $\begin{array}{l}4.59 \\
1.58 \\
1 \cdot 65 \\
2.07 \\
2.08 \\
2.41 \\
0.40 \\
0.51 \\
1.13 \\
1.03 \\
2.61 \\
1.64 \\
1 \cdot 33 \\
0 \cdot 84 \\
2 \cdot 12 \\
1 \cdot 18 \\
0.96 \\
0.52 \\
2.23 \\
2.09 \\
1.43 \\
1 \cdot 14 \\
0.98 \\
1.25 \\
3.73 \\
0.74 \\
1.09 \\
- \\
-\end{array}$ & $\begin{array}{l}72 \\
90 \\
84 \\
70 \\
89 \\
63 \\
76 \\
96 \\
82 \\
77 \\
54 \\
98 \\
90 \\
89 \\
82 \\
49 \\
90 \\
85 \\
77 \\
71 \\
60 \\
86 \\
79 \\
78 \\
63\end{array}$ & $\begin{array}{l}40 \\
58 \\
39 \\
43 \\
57 \\
= \\
= \\
= \\
= \\
= \\
= \\
= \\
= \\
= \\
37 \\
57 \\
59 \\
53 \\
47 \\
44 \\
52 \\
49 \\
67\end{array}$ & $\begin{array}{l}7 \cdot 43 \\
7 \cdot 31 \\
7 \cdot 33 \\
7 \cdot 38 \\
7 \cdot 25 \\
= \\
= \\
= \\
= \\
= \\
= \\
= \\
= \\
= \\
\overline{7} \\
7 \cdot 47 \\
7 \cdot 29 \\
7 \cdot 29 \\
7 \cdot 34 \\
= \\
\overline{7 \cdot 41} \\
7 \cdot 30 \\
7 \cdot 37 \\
7 \cdot 28\end{array}$ \\
\hline $\begin{array}{c}\text { Total: Average } \\
\text { Sigma }\end{array}$ & $\begin{array}{l}9 \cdot 4 \\
4 \cdot 6\end{array}$ & $\begin{array}{l}40 \cdot 8 \\
13 \cdot 5\end{array}$ & $\begin{array}{r}13 \cdot 0 \\
5 \cdot 8\end{array}$ & $\begin{array}{l}3.42 \\
0.95\end{array}$ & $\begin{array}{l}7 \cdot 65 \\
3 \cdot 3\end{array}$ & $\begin{array}{l}5 \cdot 21 \\
2 \cdot 5\end{array}$ & $\begin{array}{l}2.44 \\
1.5\end{array}$ & $\begin{array}{l}1.60 \\
0.96\end{array}$ & $\begin{array}{l}78 \cdot 0 \\
13 \cdot 0\end{array}$ & $\begin{array}{r}51 \cdot 1 \\
7 \cdot 3\end{array}$ & $\begin{array}{l}7 \cdot 335 \\
0 \cdot 050\end{array}$ \\
\hline
\end{tabular}

was an indirect relation between the arterial oxygen saturation and the mean artery pressure $(r=0.220, p<0.05)$. However, it was significant only in the groups with heart failure and after recovery $(r=0.304$ and $0.452, p<0.01)$, whereas this relation is not significant in controls and in compensated cor pulmonale. No correlation was found between the $\mathrm{S}_{\mathrm{a}} \mathrm{O}_{2}$ and pulmonary artery wedge pressure or pressure in the right atrium, or between the arterial oxygen saturation and blood flow ( $p$ always $>0 \cdot 10$ ).

There was no significant correlation between the cardiac output and the cardiac index and the mean right atrial pressure, the pulmonary artery or pulmonary artery wedge pressure, blood gases, and $p H$. The relation between the mean pressure in

TABLE IV

HAEMODYNAMIC AND RESPIRATORY FINDINGS IN COR PULMONALE RECOVERING FROM HEART FAILURB

\begin{tabular}{|c|c|c|c|c|c|c|c|c|c|c|c|}
\hline Case No. & $\underset{(\mathbf{m m} \cdot \mathbf{H g})}{\operatorname{mRAP}}$ & $\underset{(\mathbf{m m} \cdot \mathbf{H g})}{\mathbf{m P A P}}$ & $\underset{(\mathbf{m m} . \mathbf{H g})}{\operatorname{mPAWP}}$ & $\begin{array}{c}\text { Cardiac } \\
\text { index } \\
\left(1 . / \mathrm{min} . / \mathrm{m} \cdot .^{2}\right)\end{array}$ & $\begin{array}{l}\text { Total } \\
\text { pulm. } \\
\text { resist. } \\
\text { (units) }\end{array}$ & $\underset{\text { (units) }}{\text { PAR }}$ & $\begin{array}{l}\text { EAPR } \\
\text { (units) }\end{array}$ & $\underset{\substack{\left.\mathrm{m}^{2}{ }^{2}\right) \\
\mathrm{W}_{\mathrm{r}}}}{(\mathrm{kp} \cdot \mathrm{min} . /}$ & $\underset{(\%)}{\mathbf{S}_{\mathrm{a}} \mathrm{O}_{2}}$ & $\mid \begin{array}{c}\mathbf{P}_{\mathrm{a}} \mathbf{C O}_{2} \\
\left(\mathbf{m m} . \mathrm{Hg}_{\mathbf{g}}\right)\end{array}$ & $p H$ \\
\hline $\begin{array}{r}1 \\
2 \\
3 \\
4 \\
5 \\
6 \\
7 \\
8 \\
9 \\
10 \\
11 \\
12 \\
13 \\
14 \\
15 \\
16 \\
17 \\
18\end{array}$ & $\begin{array}{r}6 \cdot 1 \\
12.0 \\
2.5 \\
6.5 \\
2.3 \\
2.0 \\
13.8 \\
3.0 \\
9.0 \\
1.0 \\
4.0 \\
1.0 \\
5.0 \\
0.0 \\
2.0 \\
4.0 \\
5.0 \\
8.9\end{array}$ & $\begin{array}{l}24 \\
29 \\
26 \\
32 \\
49 \\
47 \\
40 \\
27 \\
30 \\
23 \\
32 \\
42 \\
31 \\
39 \\
37 \\
42 \\
29 \\
24\end{array}$ & $\begin{array}{r}5.2 \\
11.0 \\
4.0 \\
6.5 \\
5.0 \\
6.0 \\
6.6 \\
8.0 \\
7.0 \\
18.0 \\
15.0 \\
14.0 \\
10.8 \\
4.0 \\
11.5 \\
19.0 \\
11.0 \\
11.2\end{array}$ & $\begin{array}{l}5 \cdot 5 \\
1 \cdot 8 \\
4 \cdot 5 \\
2 \cdot 5 \\
2 \cdot 4 \\
2 \cdot 9 \\
2 \cdot 6 \\
3 \cdot 1 \\
2 \cdot 3 \\
3 \cdot 9 \\
5 \cdot 1 \\
4 \cdot 2 \\
2 \cdot 7 \\
7 \cdot 7 \\
3 \cdot 1 \\
4 \cdot 0 \\
4 \cdot 2 \\
3 \cdot 5\end{array}$ & $\begin{array}{r}2 \cdot 2 \\
7 \cdot 8 \\
4 \cdot 0 \\
7 \cdot 2 \\
11.5 \\
8.9 \\
8 \cdot 0 \\
5 \cdot 1 \\
7 \cdot 8 \\
4 \cdot 3 \\
3.3 \\
5 \cdot 6 \\
7.0 \\
3.1 \\
6.7 \\
6 \cdot 8 \\
4 \cdot 2 \\
3.8 \\
\end{array}$ & $\begin{array}{r}1.6 \\
4.8 \\
3.4 \\
5.7 \\
10.3 \\
7.8 \\
6.7 \\
3.6 \\
6.0 \\
0.9 \\
1.8 \\
3.7 \\
4.6 \\
2.9 \\
4.6 \\
3.7 \\
2.6 \\
2.1\end{array}$ & $\begin{array}{l}0.6 \\
3.0 \\
0.6 \\
1.5 \\
1.2 \\
1.1 \\
1.3 \\
1.5 \\
1.8 \\
3.4 \\
1.5 \\
1.9 \\
2.4 \\
0.2 \\
2.1 \\
3.1 \\
1.6 \\
1.7\end{array}$ & $\begin{array}{l}1.43 \\
0.41 \\
1.52 \\
0.93 \\
1.62 \\
1.90 \\
1.19 \\
1.08 \\
0.68 \\
1.23 \\
2.05 \\
2.53 \\
1.01 \\
4.44 \\
1.53 \\
2.18 \\
1.45 \\
0.76\end{array}$ & $\begin{array}{l}85 \\
97 \\
79 \\
93 \\
71 \\
82 \\
85 \\
90 \\
83 \\
84 \\
65 \\
84 \\
92 \\
60 \\
72 \\
87 \\
81 \\
88\end{array}$ & $\begin{array}{l}\overline{40} \\
\bar{Z} \\
\bar{Z} \\
\bar{Z} \\
\bar{Z} \\
\overline{-} \\
\overline{-} \\
35 \\
48 \\
50 \\
38 \\
63 \\
41\end{array}$ & $\begin{array}{l}\overline{7 \cdot 43} \\
= \\
= \\
= \\
= \\
= \\
= \\
\bar{Z} \\
7 \cdot 43 \\
7.36 \\
7.37 \\
7.39 \\
7.39 \\
7.35\end{array}$ \\
\hline $\begin{array}{c}\text { Total: Average } \\
\text { Sigma }\end{array}$ & $\begin{array}{l}4 \cdot 6 \\
3 \cdot 9\end{array}$ & $\begin{array}{l}33 \cdot 5 \\
8 \cdot 8\end{array}$ & $\begin{array}{l}9 \cdot 6 \\
5 \cdot 1\end{array}$ & $\begin{array}{l}3 \cdot 68 \\
1 \cdot 2\end{array}$ & $\begin{array}{l}6 \cdot 10 \\
2 \cdot 5\end{array}$ & $\begin{array}{l}4 \cdot 40 \\
2 \cdot 7\end{array}$ & $\begin{array}{l}1 \cdot 70 \\
0 \cdot 8\end{array}$ & $\begin{array}{l}1.60 \\
0.58\end{array}$ & $\begin{array}{r}81 \cdot 8 \\
9 \cdot 0\end{array}$ & $\begin{array}{r}45 \cdot 1 \\
5 \cdot 9\end{array}$ & $\begin{array}{l}7 \cdot 388 \\
0.076\end{array}$ \\
\hline
\end{tabular}


TABLE V

HAEMODYNAMIC AND BLOOD GASES IN PATIENTS WITH NORMAL AND RAISED PULMONARY WEDGE PRESSURE

\begin{tabular}{|c|c|c|c|c|c|c|c|c|}
\hline & \multicolumn{4}{|c|}{ All patients } & \multicolumn{4}{|c|}{ Heart failure } \\
\hline \multirow{2}{*}{. } & \multicolumn{2}{|c|}{ Normal (65 cases) } & \multicolumn{2}{|c|}{ High (27 cases) } & \multicolumn{2}{|c|}{ Normal (13 cases) } & \multicolumn{2}{|c|}{ High (16 cases) } \\
\hline & Average & Sigma & Average & Sigma & Average & Sigma & Average & Sigma \\
\hline $\begin{array}{l}\text { mRAP (mm.Hg) } \\
\text { mPAP (mm.Hg) } \\
\text { mPAWP (mm.Hg) } \\
\text { Cardiac index (l./min./ } \\
\text { m.2) } \\
\text { Total pulm. resist. } \\
\text { (units) } \\
\text { PAR (units) } \\
\text { EAPR (units) } \\
W_{\mathbf{r}}\left(\mathrm{kp.m.} / \mathrm{min} . / \mathrm{m}^{2}{ }^{2} \text { ) }\right. \\
\mathrm{S}_{\mathrm{a}} \mathrm{O}_{2} \text { (\%) } \\
\mathbf{P}_{\mathrm{a}} \mathrm{CO}_{2} \text { (mm.Hg) } \\
\text { pH }\end{array}$ & $\begin{array}{c}3 \cdot 9 \\
29 \cdot 8 \\
6 \cdot 3 \\
3 \cdot 7 \\
5 \cdot 24 \\
4 \cdot 11 \\
1 \cdot 13 \\
1 \cdot 36 \\
85 \cdot 8 \\
46 \cdot 2 \\
7 \cdot 373\end{array}$ & $\begin{array}{l}5 \cdot 6 \\
8 \cdot 1 \\
5 \cdot 1 \\
1 \cdot 4 \\
3 \cdot 34 \\
2 \cdot 38 \\
0 \cdot 51 \\
0 \cdot 89 \\
6 \cdot 1 \\
7 \cdot 3 \\
0 \cdot 077\end{array}$ & $\begin{array}{l}7 \cdot 9 \\
40 \cdot 1 \\
16 \cdot 6 \\
3 \cdot 7 \\
7 \cdot 31 \\
4 \cdot 30 \\
3 \cdot 01 \\
1 \cdot 73 \\
78 \cdot 4 \\
45 \cdot 1 \\
7 \cdot 352\end{array}$ & $\begin{array}{l}3 \cdot 9 \\
16 \cdot 8 \\
10 \cdot 1 \\
1 \cdot 4 \\
2 \cdot 7 \\
2 \cdot 49 \\
1.53 \\
1 \cdot 01 \\
12 \cdot 9 \\
8 \cdot 1 \\
0.092\end{array}$ & $\begin{array}{l}6 \cdot 5 \\
35 \cdot 8 \\
7 \cdot 3 \\
3 \cdot 4 \\
6 \cdot 39 \\
5 \cdot 0 \\
1 \cdot 39 \\
1 \cdot 36 \\
82 \cdot 6 \\
51 \cdot 0 \\
7 \cdot 340\end{array}$ & $\begin{array}{l}4 \cdot 6 \\
7 \cdot 2 \\
4 \cdot 1 \\
1 \cdot 5 \\
3 \cdot 6 \\
2 \cdot 75 \\
0 \cdot 50 \\
0 \cdot 91 \\
5 \cdot 7 \\
6 \cdot 6 \\
0 \cdot 086\end{array}$ & $\begin{array}{l}11 \cdot 0 \\
45 \cdot 0 \\
17 \cdot 7 \\
3 \cdot 5 \\
8 \cdot 69 \\
5 \cdot 26 \\
3 \cdot 43 \\
1 \cdot 77 \\
73 \cdot 7 \\
49 \cdot 0 \\
7 \cdot 350\end{array}$ & $\begin{array}{c}3.0 \\
15.4 \\
6.1 \\
1.3 \\
3.79 \\
3.14 \\
1.26 \\
1.04 \\
13.1 \\
11.3 \\
0.101\end{array}$ \\
\hline
\end{tabular}

the pulmonary artery and in the wedged catheter was on the borderline of statistical significance $(r=0.216, p=0.05)$. The coefficients of correlation in the clinical groups varied a great deal but were homogeneous. Extra-arterial resistance showed no significant correlation either with the total or with the arteriolar pulmonary resistances. Surprisingly, no relation was found between arterial $\mathrm{CO}_{2}$ tension or blood $\mathrm{pH}$ and any haemodynamic values.

Patients with Increased Pulmonary Artery Wedge Pressure. In controls with normal pulmonary pressure, the pulmonary artery wedge pressure

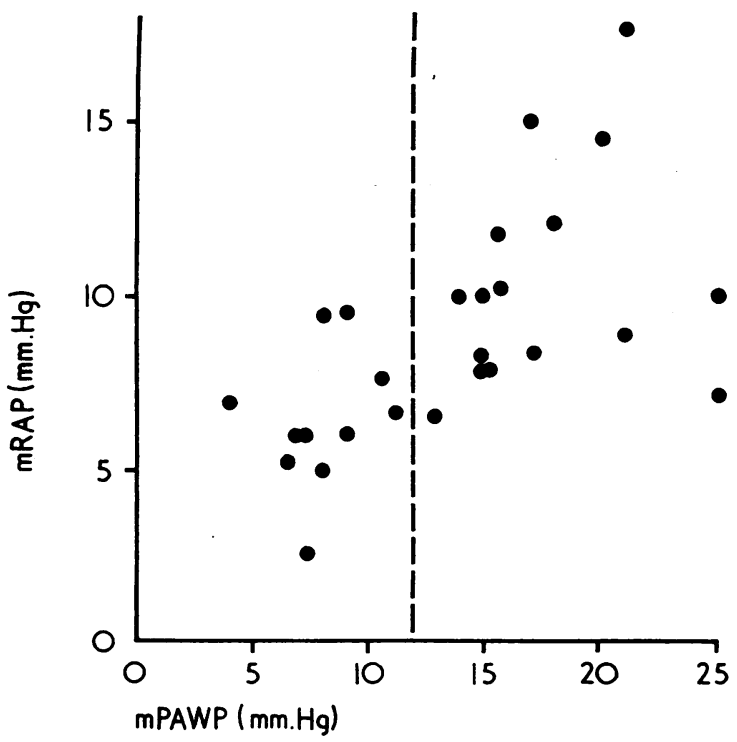

Frg.-Relation between the mean right atrial pressure and the mean pulmonary arterial wedge pressure. This relation was highly significant $(r=0.506 ; p<0.01)$. was also normal. Raised pulmonary artery wedge pressure was rare in cor pulmonale before failure $(16 \%)$ and after recovery from failure $(22 \%)$, but in cor pulmonale with heart failure this pressure was raised in 55 per cent of cases. Patients with increased pulmonary artery wedge pressure differed in several respects from other patients with cor pulmonale. (1) They had a higher pressure in the pulmonary artery $(p<0.05)$. (2) Total pulmonary resistance was higher since the average blood flow was the same in the group with normal as well as with raised pulmonary wedge pressure. As the arteriolar resistance did not differ in the two groups, the extra-arterial component was at fault (Table V). (3) The right atrial pressure was higher and there was a highly significant correlation between the pulmonary artery wedge pressure and the pressure in the right atrium (Figure). (4) In patients with increased pulmonary artery wedge pressure, the arterial oxygen saturation was significantly lower than in patients with normal wedge pressure (Table VI).

The difference between patients with increased and normal pulmonary artery wedge pressure in cor pulmonale was even more significant if only patients with heart failure were taken into consideration.

TABLE VI

HYPERTENSION AND ARTERIAL OXYGEN SATURATION

\begin{tabular}{c|c|c}
\hline $\mathrm{S}_{\mathrm{a}} \mathrm{O}_{2}(\%)$ & $\begin{array}{c}\text { No. of } \\
\text { cases }\end{array}$ & $\begin{array}{c}\text { Per cent of cases } \\
\text { with raised pulm. } \\
\text { artery wedge } \\
\text { pressure }\end{array}$ \\
\hline $\begin{array}{c}91-100 \\
81-90 \\
71-80\end{array}$ & 20 & $\begin{array}{c}15 \cdot 0 \\
<71\end{array}$ \\
$<79$ & $\begin{array}{c}24 \cdot 4 \\
26 \cdot 3 \\
75 \cdot 0\end{array}$ \\
\hline
\end{tabular}




\section{Discussion}

The comparison of respiratory and haemodynamic data in our patients shows the well-known relation between hypoxaemia and increases of pressure in the pulmonary artery; however, an indirect relation between $\mathrm{S}_{\mathrm{a}} \mathrm{O}_{2}$ and mPAP could be ascertained only in patients with low mean $\mathrm{O}_{2}$ saturation, i.e. in the group manifesting heart failure or recovering from failure (recompensated cor pulmonale).

In progressive cor pulmonale, a highly significant difference in the tension of arterial $\mathrm{CO}_{2}$ was found between the cases of compensated cor pulmonale and those with heart failure. There was, however, no statistically significant correlation of mPAP with $\mathrm{P}_{\mathrm{a}} \mathrm{CO}_{2}$ or with $\mathrm{pH}$ of the arterial blood. It is possible that the relation between $\mathrm{P}_{\mathrm{a}} \mathrm{CO}_{2}$ and mPAP in our patients is closer than would follow from the figures we obtained. In our series only 6 patients had $\mathrm{P}_{\mathrm{a}} \mathrm{CO}_{2}$ higher than $55 \mathrm{~mm} . \mathrm{Hg}$ and only 2 were higher than $60 \mathrm{~mm} . \mathrm{Hg}$. In acute hypercapnic acidosis produced by breathing $\mathrm{CO}_{2}$ mixtures, the relation of $\triangle \mathrm{P}_{\mathrm{a}} \mathrm{CO}_{2}$ to $\triangle \mathrm{mPAP}$ was significant (Herles, Ježek, and Boudík, 1966).

Increased pulmonary artery wedge pressure in cor pulmonale has been recorded by many authors but has not received due attention. It was ascribed usually to complicating failure of the left heart, i.e. to the simultaneous presence of ischaemic heart disease. However, in most instances the increase in wedge pressure cannot be explained in this way (Herles, Daum, and Bednár, 1960), since in such cases at necropsy no changes in the left side of the heart and no signs of pulmonary congestion have been found. We assume that pulmonary artery wedge pressure belongs to the rearrangement of pulmonary circulation in certain stages of chronic cor pulmonale.

A number of factors contribute to the pulmonary artery wedge pressure: (1) pressure in the left atrium; (2) vasoconstriction, or increased capillary, or venular resistance elicited in other ways; (3) increased blood flow through bronchopulmonary anastomoses, both arterial and venous.

The possibility of increases in pressure in the left atrium in uncomplicated cor pulmonale cannot be denied. It need not be a consequence of insufficiency of the left ventricle, but it might be a result of a diminished compliance of the left ventricle in the presence of hypertrophy of the right ventricle, if the blood flow to the left side of the heart is increased. In some cases of cor pulmonale in chronic bronchitis we did find increased pressure in the left atrium (Ježek, 1967). Nevertheless, this cannot be the only factor of increased post-arterial resistance and of increase in pressure in the wedged pulmonary artery, since we were able to find differences in pressure at different sites in the lung which could not be explained by hydrostatic influences (Herles and Ježek, 1967). The experimental work of von Euler and Liljestrand (1946), Nisell (1948), and others suggested that the post-arteriolar resistance might be increased by constriction of the pulmonary venules. The high incidence of advanced venulosclerosis in chronic cor pulmonale suggests that the site of increased vascular resistance might also be sought there.

In considering the genesis of increased pressure in the wedged pulmonary artery, the bronchopulmonary collaterals deserve special attention. Pathologists (Hayek, 1951; Cudkowicz and Armstrong, 1953; Bednár and Dobiáš, 1965; Liebow, 1953, 1954) have shown that arterial bronchopulmonary anastomoses are common in emphysema. In chronic bronchitis with emphysema all branches of the pulmonary artery can easily be filled up from the bronchial arteries, in contradistinction to the normal lung or to other lung diseases (Dobiás, 1965). On wedging the catheter into the pulmonary artery, the circulation in the terminal part of the pulmonary branches can be maintained by the bronchial arteries. In cases of increased systemic venous pressure, such as in right ventricular failure in cor pulmonale, bronchial veins may carry systemic blood into pulmonary veins (Liebow, 1953, 1954).

Significant correlation between the raised pressure in the right atrium and in the wedged pulmonary artery in progressive cor pulmonale with heart failure supports this concept. If the venules constrict or their extensibility is curtailed, the wedge pressure may increase. When explaining the enlargement of the left ventricle in some cases of emphysema, increased blood flow from bronchopulmonary anastomoses into the left heart and left-to-right circulatory shunt bypassing the right heart have been assumed in the past (Kountz, Alexander, and Prinzmetal, 1936) and more recently (Fluck, Chandrasekar, and Gardner, 1966). The increased filling of the left heart may raise the end-diastolic pressure in the left ventricle as well as the pulmonary artery wedge pressure.

Statistical and clinical studies to date do not explain the mechanism of increase of pressure in the pulmonary artery in chronic cor pulmonale. Our findings, however, show that the site of pulmonary resistance in cor pulmonale in chronic bronchitis must be sought also in the post-arteriolar sector of the pulmonary circulation. Conspicuous hypoxaemia and respiratory acidosis; which accompany heart failure and which do not completely 
recede with clinical recovery, may bring about this change in post-arteriolar resistance. The significant changes in acute respiratory acidosis evoked in men by inhalation of air mixture with $\mathrm{CO}_{2}$ (Fishman, Fritts, and Cournand, 1960; Herles et al., 1966) as well as the animal experiments with acute non-respiratory acidosis (Bergofsky et al., 1961; Harvey et al., 1951) support this.

\section{SUMMARY}

In examining the haemodynamics of chronic bronchitis, we frequently came across cases with the post-arteriolar type of pulmonary hypertension, i.e. with pulmonary artery wedge pressure of 12 $\mathrm{mm} . \mathrm{Hg}$ or higher, where no overt clinical changes in the function or anatomy of the left heart were found. In the effort to assess the incidence of raised pulmonary artery wedge pressure in cor pulmonale and to elucidate its origin, we analysed haemodynamic and respiratory data in 121 patients with chronic obstructive bronchitis. The patients without pulmonary hypertension were compared with those with cor pulmonale, i.e. with those whose pulmonary artery mean pressure exceeded 20 mm.Hg. In uncomplicated chronic bronchitis the pulmonary artery wedge pressure was never raised. In cor pulmonale it was found to be higher in 20 per cent before and after recovery from failure, and it was raised in more than half of the patients with heart failure. A significant relation was found between the pulmonary artery wedge pressure and the pulmonary artery pressure, the pressure in the right atrium, and hypoxaemia. Pulmonary hypertension with high pulmonary artery wedge pressure seems to form a particular haemodynamic type of progressive cor pulmonale in chronic bronchitis. It is assumed that increased post-arteriolar resistance plays a major part in the development of pulmonary hypertension in chronic obstructive bronchitis.

\section{REFERENCES}

Bednáŕ, B., and Dobiáš, J. (1965). Das dystelektdtische Lungenemphysem. Virchows Arch. path. Anat., 340, 53.

Bergofsky, E. H., Lehr, D. E., Tuller, M. A., Rigatto, M., and Fishman, A. P. (1961). The effects of acute alkalosis and acidosis on the pulmonary circulation. Ann. N.Y. Acad. Sci., 92, 627.

Cudkowicz, L., and Armstrong, J. B. (1953). The bronchial arteries in pulmonary emphysema. Thorax, 8, 46 .

Dobiás, J. (1965). Morphology of some rather important lesions of pulmonary vessels. (In Czech.) Acta. Univ. Carol. Med. (Praha), 11, 529.

von Euler, U. S., and Liljestrand, G. (1946). Observations on the pulmonary arterial blood pressure in the cat. Acta physiol. scand., 12, 301.

Fishman, A. P., Fritts, H. W., Jr., and Cournand, A. (1960). Effects of breathing carbon dioxide upon the pulmonary circulation. Circulation, 22, 220.

Fluck, D. C., Chandrasekar, R. G., and Gardner, F. V. (1966). Left ventricular hypertrophy in chronic bronchitis. Brit. Heart f., 28, 92.

Harvey, R. M., Ferrer, M. I., Richards, D. W., Jr., and Cournand, A. (1951). Influence of chronic pulmonary disease on the heart and circulation. Amer. F. Med., 10, 719.

Hayek, H. von. (1951). Über die funktionelle Anatomie der Lungengefässe. Verh. disch. Ges. Kreisl.-Forsch., 17, 17.

Herles, F. (1966). The pulmonary artery wedge pressure. Cor et vasa (Praha), 8, 161.

-, Daum, S., and Bednári, B. (1960). The cardiopulmonary failure with elevated wedge pressure in pulmonary emphysema. Acta tertii Europaei de Cordis Scientia Conventus, Roma, Pars Altera, A, p. 491.

-, and Ježek, V. (1967). The factors influencing the pulmonary wedge pressure. Acta cardiol. (Brux.)., 22, 575.

$\longrightarrow,-$, and Boudik, F. (1966). The influence of acute and chronic respiratory acidosis upon the pulmonary circulation. Bull. Physio-path. resp., 2, 459.

Ježek, V. (1967). Round table discussion. Entretiens de Physio-pathologie Respiratoire, Nancy, September 1967.

Kountz, W. B., Alexander, H. L., and Prinzmetal, M. (1936). The heart in emphysema. Amer. Heart f., 11, 163.

Králová, L., Ježek, V., Linhartová, J., and Stěpánek, J. (1965). Incidence of ischaemic heart disease in cor pulmonale and diagnostic possibilities. (In Czech.). Čas. Lék. čes., 104, 654.

Liebow, A. A. (1953). The bronchopulmonary venous collateral circulation with special reference to emphysema. Amer. F. Path., 29, 251.

(1954). Some aspects of collateral circulation of the lung. Bull. N.Y. Acad. Med., 30, 66.

Nisell, O. (1948). Effects of oxygen and carbon dioxide on the circulation of isolated and perfused lungs of the cat. Acta physiol. scand., 16, 121.

Pearson and Spearman. Quoted by Roth, Z., Josifko, M. Maly, V., and Trcke, V. (1962). Statistical Methods in Experimental Medicine (in Czech). SZdN, Prague.

Van Slyke, D. D., and Neill, J. M. (1924). The determination of gases in blood and other solutions by vacuum extraction and manometric measurement. $\mathcal{f}$. biol. Chem., 61, 523. 